• 研究报告・

\title{
木棉黄花个体的适应意义
}

\author{
向文倩 任明迅 ${ }^{*}$ \\ (海南大学生态与环境学院环南海陆域生物多样性研究中心, 海口 570228)
}

\begin{abstract}
摘要: 木棉(Bombax ceiba) 花大、红色, 多为鸟媒传粉, 但也存在频率极低的黄花个体, 有蜜蜂访花。本文对比研 究了海南岛木棉红花、黄花的花部特征及传粉过程, 以解释黄花个体的适应意义。木棉两种花色表型的花蜜组分 (葡萄糖和果糖)及挥发性物质(烷烃类、酯类、酚类等)没有显著差异, 但黄花花瓣的反射波长范围均在鸟类与蜜蜂 视觉范围内, 可同时吸引鸟和蜜蜂。木棉黄花的雌雄异熟程度较小(花粉活性时期与柱头可受期重叠较大), 可在传 粉媒介访花次数有限的情况下同时输出或接受花粉。另一方面, 黄花有着更大程度的柱头探出式雌雄异位, 降低 了较小程度雌雄异熟可能导致的自交与雌雄功能干扰。木棉存在明显的花粉限制, 且黄花的坐果率 $(1.08 \pm 0.56) \%$ 显著低于红花 $(3.27 \pm 0.93) \%$ 。我们推测, 黄花个体可能通过增加木棉访花者类群多样性、降低䧳雄异熟程度而提 高了木棉的繁殖成功率, 也通过吸引蜜蜂而降低了蜜蜂对红花鸟媒传粉的干扰。
\end{abstract}

关键词：鸟媒传粉; 性别干扰; 异交授粉; 雌雄异熟; 雌雄异位

\section{Adaptive significance of yellow flowered Bombax ceiba (Malvaceae)}

\author{
Wenqian Xiang, Mingxun Ren ${ }^{*}$ \\ Center for Terrestrial Biodiversity of the South China Sea, College of Ecology and Environment, Hainan University, Haikou \\ 570228
}

\begin{abstract}
Bombax ceiba is a tall tree species with predominantly red flowers and is normally pollinated by birds. In some populations, a yellow flowered variety occurs. Honeybees frequently visit these uncommon yellow flowers but how this adaptation affects the life history of this variant remains unexplored. In the present study, floral syndrome and pollination mechanism of yellow flowers were compared with red flowers of B. ceiba populations on Hainan Island, southern China. The results showed that main nectar components of two floral phenotypes are both glucose and fructose dominated, and the volatile chemicals are mainly alkanes, esters, phenols and acids with no significant difference between the two phenotypes. The relative spectral reflectance showed that both birds and honeybees could detect the yellow flowers, suggesting visitation by honeybees can compensate for times when bird visitation is low. Compared with red flowers, yellow flower had a lower degree of dichogamy, which might increase selfing possibilities and provide reproduction assurance when red flowers receive low bird visitation. Interestingly, yellow flowers had a relatively higher degree of herkogamy, i.e. stigmas are much higher than anthers when compared to the red flowers. Greater herkogamy in yellow flowers may reflect an adaptation to decreased dichogamy, avoiding autonomous selfing and interference between female and male organs. Fruit set of the yellow phenotype $(1.08 \pm 0.56) \%$ was lower than that of red phenotype $(3.27 \pm 0.93) \%$, suggesting pollen-limitation in $B$. ceiba. We propose that yellow flowers, with greater herkogamy but lower dichogamy, promote pollination via attracting diverse pollinators and protect red flowers from disturbance of honeybees.
\end{abstract}

Key words: bird-pollination; sexual interference; reproductive assurance; dichogamy; herkogamy

花冠颜色是动物传粉者进行花朵定位最重要 的视觉信号之一(Chittka \& Waser, 1997)。不同的传 粉者往往对不同花色有着不同的敏感度, 从而表现
出对某种花色的访问倾向性，如鸟类与蝶类多访问 红花、蜂类偏好黄花和蓝紫花(Chang \& Rausher, 1999; Fenster et al, 2004; 张大勇, 2004)。 
同一种植物的不同个体也会表现出花色多态 性(Stanton et al, 1986; Schoonhoven et al, 2007; Majetic et al, 2009; Koski \& Ashman, 2016), 可能具 有不一样的适应意义(Vaidya et al, 2018)。在红木 (Bixa orellana)中, 红花与紫花的花药多、雌雄异位 程度大, 较白色或钴紫色个体的异交率和结实率更 高 (Joseph \& Siril, 2013)。圆叶牵牛 (Ipomoea purpurea)可能通过近交衰退的压力来维持其花色 多态性(Chang \& Rausher, 1999); 萝卜(Raphanus sativus)通过两种花色分别提高雌雄功能(Stanton et $\mathrm{al}, 1986)$, 也减少了植食动物的采食(Irwin, 2003)。

同种植物的不同花色在种群内的分布频率有 时极不均衡。如根爪兰(Dactylorhiza sambucina)除 了常见的黄花和紫花个体, 还有分布频率极低(约 占种群大小的 $10 \%-20 \%$ ) 的粉花个体 (Gigord, 2001)。这些极低频率的粉花个体可能通过增加花色 多态性，提高和延长了无报酬的根爪兰对传粉者的 吸引力 (Gigord, 2001)。十字花科自花授粉的 Boechera stricta白花个体易受植食动物取食(Vaidya et al, 2018)。在水分充足的环境中, 白花个体占绝对 优势, 紫花个体稀少甚至消失; 但在干旱、酸性土 壤等恶劣环境下, 紫花个体分布频率可以达到 $80 \%$ 。这种花色比例的反转, 主要是因为紫花个体 体内化学物质能提高植物耐受性和避开植食动物 取食(Vaidya et al, 2018)。这些研究表明, 频率低的 花色个体可能具有该物种常见花色所没有的适应 意义, 增强了物种在复杂多变环境中的适应能力。

木棉(Bombax ceiba)是锦葵科木棉亚科(APG II, 2003)多年生落叶大乔木, 主要分布在热带与亚热 带亚洲的干热河谷、路旁和开阔地域(Barwick, 2004; Zhou et al, 2015)。花期 3-4月, 雄荵群分内、外两轮, 外轮雄荵花丝基部合生; 内轮又分为内轮长雄荵 (花丝大部分合生)、内轮短雄荵(花丝基部合生) (Davis \& Mariamma, 1965; 任明迅, 个人观察)。木 棉的花通常为深红色 (工业国际标准色卡 RAL3020)、桔红(RAL2002)、橙色(RAL2008), 鸟媒 传粉(Aluri et al, 2005), 但在中国云南、广西及海南 岛的部分种群中出现了较低频率的黄花(RAL1016) 表型 ${ }^{\mathbb{1}}$, 有大量蜜蜂访花, 能自然结实(向文倩, 个

(1) 韦俏俏 (2017) 崇左市木棉资源调查与开发利用价值的评估. 硕士 学位论文, 广西大学, 南宁.
人观察)。根据前期实验的花瓣反射波谱分析，木棉 的深红色、桔红、橙色花瓣反射波谱基本一致(向文 倩和任明迅，未发表数据)。因此，本文将木棉的深 红色、桔红、橙色花均视为“红花”，与黄花形成分 布频率和反射光谱截然不同的两类表型。本文通过 对比分析海南岛木棉两种花色表型的花部综合征、 自然传粉过程及繁育系统等, 探讨黄花与红花表型 可能的相互作用及其对木棉繁殖的影响, 以解释黄 花的适应意义及其低频率发生的机制。

\section{研究地点}

研究地位于海南岛西部昌江县霸王岭国家级 自然保护区外围的霸王镇宝山村 $\left(18^{\circ} 53^{\prime}-19^{\circ} 30^{\prime} \mathrm{N}\right.$, $\left.108^{\circ} 38^{\prime}-109^{\circ} 17^{\prime} \mathrm{E}\right)$ 。这里是海南岛木棉最为集中分 布的区域，木棉连绵分布于山沟旁、路边及田坎附 近。本研究区域木棉成年植株约 1,000 株, 黄花个体 5 株，不到种群大小的 $1 \%$ 。花期 $2-3$ 月。

\section{研究方法}

\section{1 花部综合征与开花进程}

在2017年和2018年3月上旬的木棉盛花期，选 择红花木棉5株、黄花木棉5株, 分别取刚开放的红 花和黄花各35朵，用精确度为 $1 \mathrm{~mm}$ 的直尺测量内 轮长雄荵、内轮短雄荵和外轮雄芯的花药大小、花 丝长度和雌雄异位 (柱头与最靠近的雄芯花药的空 间距离)等。

标记刚打开的红花、黄花各20朵，每隔6 h分别 取外轮雄芯、内轮长雄荵、内轮短雄荵的少许花粉 于载玻片上，滴1 $\mu \mathrm{L}$ 浓度 $1 \%$ 的噻唑蓝(MTT)溶液, 室温下静置10 min后用光学显微镜观察, 花粉被染 成蓝紫色表示花粉有活力(Rodrigue-Riano \& Dafni, 2000)。每隔 $6 \mathrm{~h}$ 取木棉红花、黄花表型的柱头，用联 苯胺-过氧化氢反应液染色10 min (Dafni \& Maués, 1998)。蓝色且产生大量气泡的柱头为可受柱头(有 活性), 无气泡或气泡很少的柱头没有活性(Dafni \& Maués, 1998)。

取刚开放的红花、黄花各 10 朵，用便携式光谱 仪(赛曼S3000-UV-NIR)和光纤反射探针 (Fiber Y $400 \mu \mathrm{m})$ 测量两种表型的花瓣反射率。光源采用脉冲 氙灯(Xe-02), 参考光使用标准反射白板(WB-02), 木棉嫩叶作为背景光参照物。测量时将探针倾斜 $45^{\circ}$ 
并置于距离花瓣表面 $5 \mathrm{~mm}$ 处。用SpectraProV3.3 (杭 州塞曼科技有限公司)整理反射率数据。

取刚开放的红花、黄花各5朵, 测量开花第一天 的花蜜体积; 用移液器吸取两种表型花蜜各 $25 \mu \mathrm{L}$ 带 回实验室, 加入等量超纯水溶解, 干燥后加入 $1 \mathrm{~mL}$ 丙酩(色谱纯)溶解, 用 $0.45 \mu \mathrm{m}$ 过滤膜过滤后用气相 色谱-质谱(GC-MS)分析测量挥发性物质及其含量(李 庆良等, 2012); 剩余花蜜用高效液相色谱(HPLC)分 析测量其糖组分及含量(Grunfeld et al, 1989)。

\section{2 繁育系统}

\subsection{1 异交指数}

通过花直径、雌雄芯空间位置与成熟顺序等, 分别对木棉红花和黄花表型进行异交指数的评判。 花直径 $<1 \mathrm{~mm}$ 记为 $0,1-2 \mathrm{~mm}$ 记为 $1,2-6 \mathrm{~mm}$ 记为 $2,>6 \mathrm{~mm}$ 记为 3 ; 雌雄荵同时成熟或雌荵先熟记为 0 , 雄荵先熟记为 1 ; 柱头和花药等高记为 0 , 雌雄异位 记为 1 ; 三者之和为异交指数。异交指数越大, 植物 的异交程度越大, 越需要传粉者(Dafni, 1992)。

\subsection{2 花粉/胚珠比}

取木棉红花、黄花即将开放而花药尚未开裂的 成熟花蕾各30朵, 迅速带回实验室。在体式显微镜 下统计花粉粒数量、胚珠数量(Dafni, 1992), 花粉数 量与肧珠数量之比即为花粉/肧珠比 $(\mathrm{P} / \mathrm{O}$ 比)。较高 的 $\mathrm{P} / \mathrm{O}$ 值代表植物以异交为主, 较低的 $\mathrm{P} / \mathrm{O}$ 值代表以 自交为主(Cruden, 1977)。

\section{3 传粉观察与坐果率统计}

根据龚燕兵和黄双全(2007)的方法, 选择木棉 红花植株、黄花植株各 5 个个体, 在晴朗天气的 7:00-8:00、11:00-12:00和17:00-18:00, 连续7天观察 访花者类型与访花行为。

记录监测植株的单株总开花数(开花早期、盛花 期、末期的花朵数量之和)。待 4 月中旬完全坐果后, 统计果实数。每个植株果实数占总开花数的百分率, 即为该植株的坐果率。

\section{4 数据分析}

采用SPSS 25.0统计软件的t-检验, 对木棉两种 花色个体(因子)三种雄荵长度、花蜜产量、花蜜糖 组分、坐果率等(因变量)进行显著性分析; 用 One-Way ANOVA分析花内三种雄芯(因子)的花药 数量、花药大小、花粉粒数量等(因变量)有无显著 差异进行检测。

\section{结果}

\section{1 花部综合征}

\subsection{1 花部特征}

木棉花簇生枝顶，花辐射对称、碗状、肉质。 柱头 5 分叉, 开花之初柱头合拢直立, 后逐渐向外 伸展。雄芯群基部合生，形成围绕子房和花柱基部 短的雄荵管。雄荵群分成内、外两轮, 外轮雄芯分 散, 花丝基部合生成5束，每束10-15枚雄荵; 内轮 雄荵群围绕花柱簇生在一起, 10枚, 花丝两两大部 分合生, 仅花药叉开; 内轮短雄荵位于内轮长雄芯 外侧, 与内轮长雄荵间隔排列, 多为 10 枚, 花丝常 两两基部合生。

花部特征统计量用SPSS软件中P-P图、Q-Q图 检测, 各点近似围绕着直线, 均满足正态分布。t-检验 中, $F>0.05$, One-Way ANOVA方差齐性显著性大于 0.05 , 均满足方差齐性。木棉内轮长雄荵花药长度 约是内轮短雄荵、外轮雄荵花药长度的 2 倍; 外轮雄 芯花药数量约是内轮长雄荵、内轮短雄荵的 6 倍; 外 轮雄荵花粉粒数约是内轮长雄芯花粉粒数的 2 倍、 内轮短雄荵的4倍(图1) $(P<0.01)$ 。

\subsection{2 雌雄异熟和雌雄异位}

木棉花常在凌晨开放, 花后约 $6 \mathrm{~h}$ 花瓣完全展 开, 露出雌雄荵。雄性先熟, 花刚打开即具有较高 的花粉活性(图2)。花药纵裂，雄性阶段可持续约30 $\mathrm{h}$ 。木棉雌荵 5 枚分叉, 逐渐伸展, 柱头可受期可持 续4-5 d。花调谢时, 花蔩、花冠和雄芯一起脱落。

木棉红花、黄花的雌荵都高于雄荵, 属柱头探 出式雌雄异位(approach herkogamy) (Webb \& Lloyd, 1986; 张大勇, 2004)。但黄花䧳雄异位程度 $(1.61 \pm$ $0.15 \mathrm{~cm})$ 显著大于红花 $(1.34 \pm 0.18 \mathrm{~cm})(P<0.05)$ (图1)。

木棉红花䧳雄异熟程度高，柱头在花后约 $18 \mathrm{~h}$ 才开始有可受性(图2), 此时花粉活性已开始逐渐 降低。开花后30 h左右，花粉活性已经很低，而柱 头活性显著增强。花后54 h时, 花药已无残留花粉 粒，此时柱头活性最高，并可持续约 $24 \mathrm{~h}$ 。木棉的 黄花在雌雄异熟程度方面与红花表现出较大的不 同(图2): 花开放6-12 h后柱头就已伸展，并具活性， 在30 h内柱头活性达到最高。因此, 黄花的雌性功 能与雄性功能的重叠程度较大(图2)、雌雄异熟程 度较弱。 

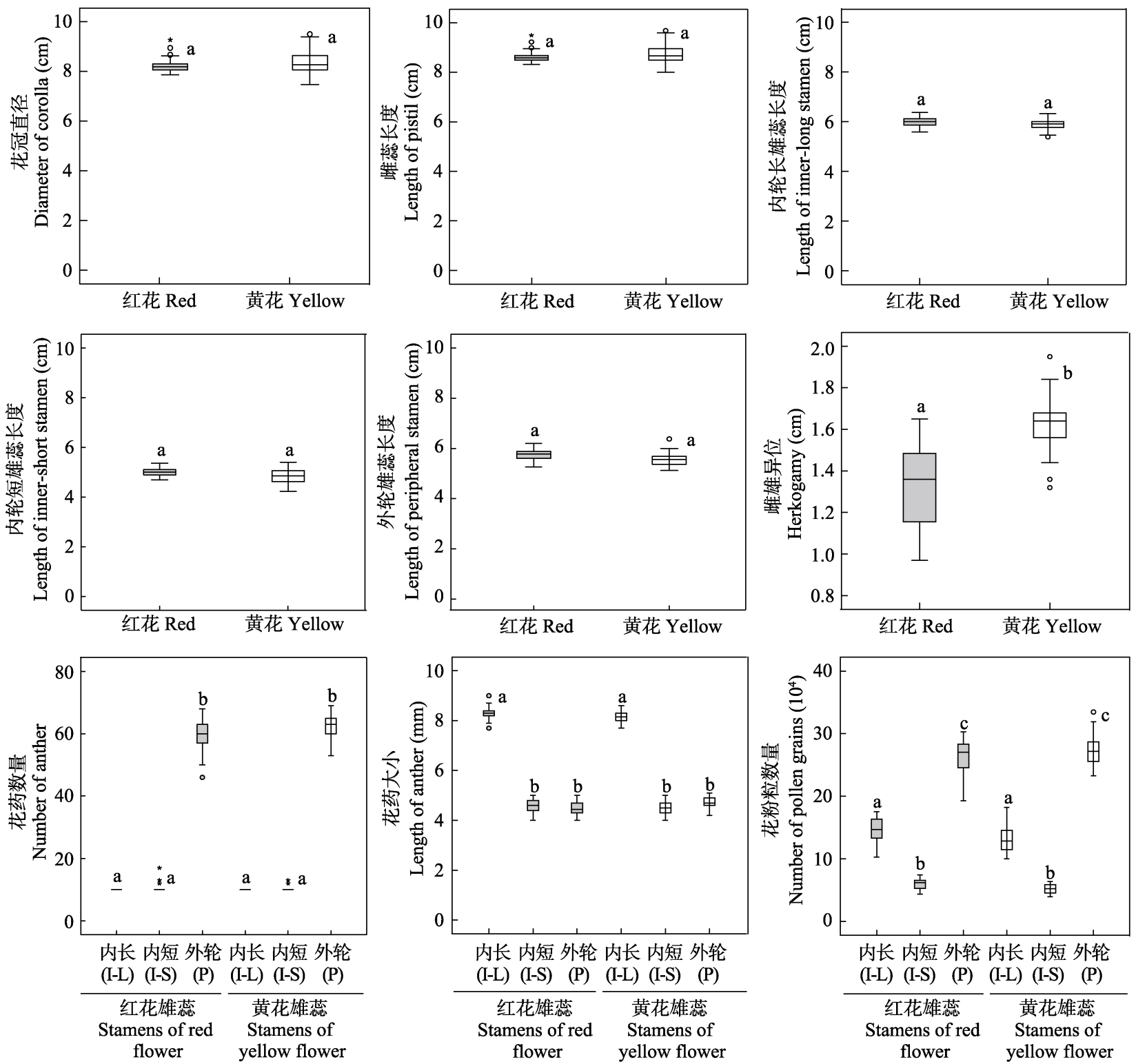

图1 木棉红花、黄花个体的花部综合征(I-L、I-S、P分别代表内轮长雄荵、内轮短雄荵、外轮雄荵心)

Fig. 1 Floral syndromes of red and yellow flowers of Bombax ceiba (I-L, I-S, P represent inner long stamen, inner short stamen and peripheral stamen, respectively)

\subsection{3 花瓣反射波谱}

花瓣反射率结果显示, 木棉红花反射波长范围 集中在580-700 nm, 黄花反射率则在 $330 \mathrm{~nm} 、 550 \mathrm{~nm}$ 处分别有个波峰, 表明红花主要吸引鸟类, 而黄花 可同时被鸟和膜翅目昆虫感知(图3)。

\subsection{4 花蜜}

木棉分泌物为无色透明的花蜜。单花花蜜产量 丰富, 红花约7.46 $\pm 1.05 \mathrm{~mL}$, 黄花约 $7.78 \pm 0.84 \mathrm{~mL}$ 。 花蜜中含有葡萄糖和果糖, 葡萄糖的含量约是果糖 的2倍(表1), 不含蔗糖; 花蜜挥发物质以烷烃类为
主，红花占(67.42 \pm 13.92)\%，黄花约占(54.75 \pm $7.78) \%(P=0.85)$ 。

木棉红花、黄花个体的花蜜产量 $(P=0.65)$ 、果 糖含量 $(P=0.91)$ 、葡萄糖含量 $(P=0.27)$, 花蜜挥发 性物质烷烃类、脂类 $(P=0.70)$ 、酚类 $(P=0.21)$ 、酸 类 $(P=0.78)$ 均无显著差异。

\section{2 繁育系统}

\subsection{1 异交指数}

木棉红花和黄花表型的花直径均可达 $9 \mathrm{~cm}$, 存 在明显的雌雄异位和雄荵先熟的雌雄异熟, 红花与 


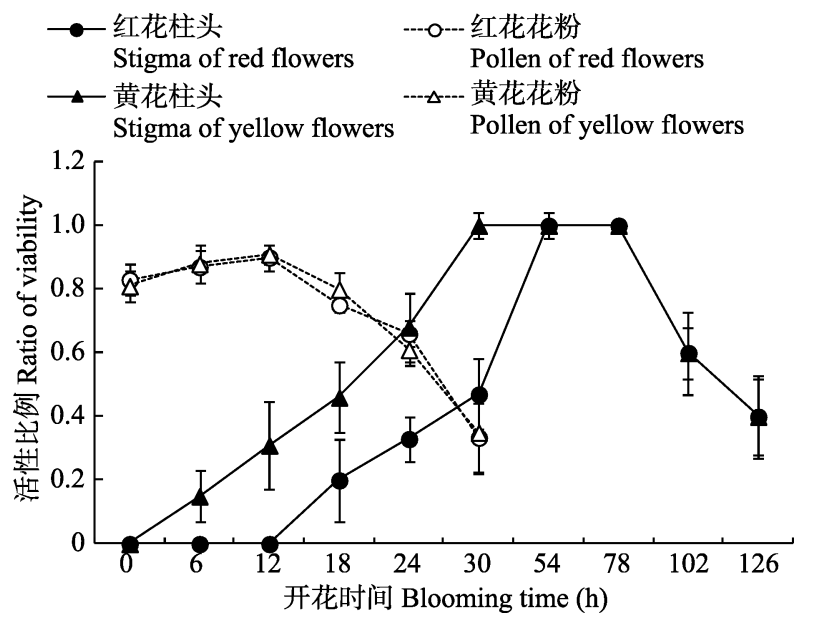

图2 木棉红花和黄花个体的花粉与柱头活力变化

Fig. 2 Pollen viability and stigma receptivity of red and yellow flowers of Bombax ceiba

黄花表型的异交指数均为5。根据Dafni (1992)的标 准, 木棉繁育系统为异交, 传粉过程依赖传粉者。

\subsection{2 性别分配}

木棉单花花粉粒数量 $(P=0.15)$ 与胨珠数量 $(P=$ 0.49)在木棉两种花色个体间无显著差异。红花表型 单花花粉数量为 $4,704,000 \pm 629,000$ 个, 黄花表型 为4,620,000 $\pm 907,000$ 个; 红花表型花粉胚珠比 $(\mathrm{P} / \mathrm{O})$ 为7,351.03 \pm 534.13, 黄花表型为 7,425.68 \pm
356.72。根据Cruden (1977)的评价标准, 木棉繁育系 统为异花授粉。

\subsection{3 访花动物与坐果率}

实验期间共进行了 7 天 $21 \mathrm{~h}$ 的传粉观察。木棉访 花动物有鸟类、蜜蜂、蝇类、松鼠等。访花动物多 取食花蜜，也取食花粉或花瓣等。其中，鸟类和蜜 蜂的访问频率最高。

鸟类同时访问红花和黄花, 早晨和傍晚访问频 率较高。盛花期时，每小时约有35只鸟类访问黄花、 79只访问红花木棉; 大多数鸟类成群(约15只)访问, 在同一朵花上停留5-40 s, 通常连续访问 2-4朵花。

蜜蜂优先访问黄花, 每小时约360次; 也访问红 花约100.4次/h (表2)。全天均有访问，早晨访问频率 最高。蜜蜂常成群结队掠夺花粉, 有时吸取花蜜。通 常一朵刚开始散粉的黄花能同时被2-8只蜜蜂访问。

木棉在自然环境下坐果率低(表2)。黄花坐果率 约(1.08 \pm 0.56$) \%$ ，显著低于红花(3.27 \pm 0.93$) \%(P<$ $0.013)$ 。

\section{讨论}

\section{1 雄荵合生与分化}

传粉观察发现，鸟类或松鼠取食花蜜与花粉时,

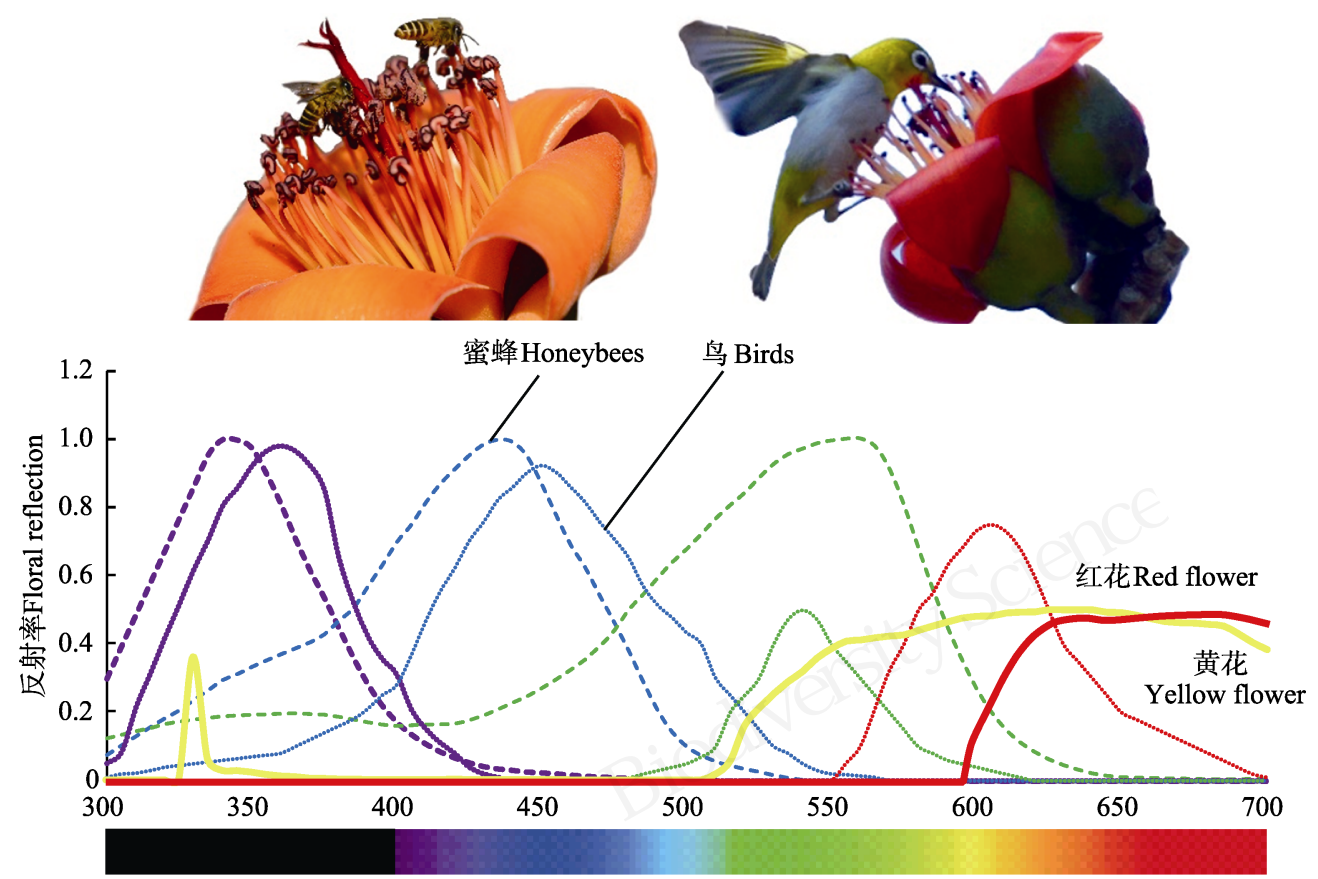

图3 木棉红花和黄花花瓣的反射光谱。短虚线为蜜蜂视觉系统, 圆点虚线为鸟类视觉系统。

Fig. 3 Relative spectral reflectance of red and yellow flowers of Bombax ceiba. The short dashed lines are spectral sensitivities of bees, the dotted line are spectral sensitivities of birds. 
表1 木棉红花与黄花的花蜜产量、主要成分与挥发性物质 含量(\%)。不同小写字母表示各处理间差异显著, $\boldsymbol{P}<\mathbf{0 . 0 5}$ 。 Table 1 Nectar volume, nectar components and volatile substances (\%) of red and yellow flowers of Bombax ceiba. Data are mean \pm standard. Different small letters mean significant difference at 0.05 level among treatments.

\begin{tabular}{lll}
\hline & $\begin{array}{l}\text { 红花 } \\
\text { Red flower }\end{array}$ & $\begin{array}{l}\text { 黄花 } \\
\text { Yellow flower }\end{array}$ \\
\hline 花蜜产量 Nectar volume (mL) & $7.46 \pm 1.05^{\mathrm{a}}$ & $7.78 \pm 0.84^{\mathrm{a}}$ \\
果糖 Fructose (mg/mL) & $13.30 \pm 1.89^{\mathrm{a}}$ & $13.09 \pm 2.79^{\mathrm{a}}$ \\
葡萄糖 Glucose (mg/mL) & $26.71 \pm 0.86^{\mathrm{a}}$ & $28.25 \pm 2.04^{\mathrm{a}}$ \\
烷烃类 Hydrocarbon (\%) & $67.42 \pm 13.92^{\mathrm{a}}$ & $54.75 \pm 7.78^{\mathrm{a}}$ \\
脂类 Lipids (\%) & $6.59 \pm 0.54^{\mathrm{a}}$ & $7.08 \pm 0.98^{\mathrm{a}}$ \\
2,4-二叔丁基苯酚 & $5.88 \pm 1.53^{\mathrm{a}}$ & $6.42 \pm 0.93^{\mathrm{a}}$ \\
2,4-Di-tert-butylphenol (\%) & & \\
$\begin{array}{l}\text { 5-羟基-2,4-二叔丁基苯基酯戊酸 } \\
\text { 5-hydroxy-pentanoic acid (\% ) }\end{array}$ & $0.79 \pm 0.09^{\mathrm{a}}$ & $0.55 \pm 0.11^{\mathrm{a}}$ \\
\hline
\end{tabular}

表2 木棉红花与黄花的访问率与坐果率(\%)。不同小写字母 表示各处理间差异显著, $\boldsymbol{P}<\mathbf{0 . 0 5}$ 。

Table 2 Pollinator visitation and fruit set (\%) of red and yellow flowers of Bombax ceiba. Data are mean \pm standard. Different small letters mean significant difference at 0.05 level among treatments.

\begin{tabular}{lll}
\hline & $\begin{array}{l}\text { 红花植株 } \\
\text { Red-flower } \\
\text { individual }(n=5)\end{array}$ & $\begin{array}{l}\text { 黄花植株 } \\
\text { Yellow-flower } \\
\text { individual }(n=5)\end{array}$ \\
\hline $\begin{array}{l}\text { 花朵数量 } \\
\text { No. of flowers per tree }\end{array}$ & $10,050 \pm 4,452.25^{\mathrm{a}}$ & $9,780 \pm 2,031.50^{\mathrm{a}}$ \\
$\begin{array}{l}\text { 每小时鸟类访花次数 } \\
\text { Bird visits per hour }\end{array}$ & $79 \pm 14.27^{\mathrm{a}}$ & $34.80 \pm 19.61^{\mathrm{b}}$ \\
$\begin{array}{l}\text { 每小时蜜蜂访花次数 } \\
\text { Honeybee visits per hour }\end{array}$ & $100.40 \pm 63.79^{\mathrm{a}}$ & $360 \pm 114.67^{\mathrm{b}}$ \\
$\begin{array}{l}\text { 果实数量 } \\
\text { No. of fruits per tree }\end{array}$ & $362 \pm 102.32^{\mathrm{a}}$ & $186.20 \pm 66.52^{\mathrm{b}}$ \\
坐果率 Fruit set (\%) & $3.27 \pm 0.93^{\mathrm{a}}$ & $1.08 \pm 0.56^{\mathrm{b}}$ \\
\hline
\end{tabular}

常站在肉质花瓣、外轮或内轮雄荵群上, 将整个头 或半个身子深入花内(图3)。这一过程中，鸟类的重 量主要压在花瓣及雄荵群合生形成的雄荵管上。木 棉雄荵花丝粗大, 且合生形成粗壮的雄荵管上, 能 承受鸟类的重量并对子房起一定的保护作用(任明 迅, 2008)。

木棉的外轮雄萝群远离花柱和内轮雄萝群, 降 低了雌雄功能干扰(张大勇, 2004; 任明迅，2009)。 另一方面, 木棉内轮长雄荵花丝两两大部分合生, 与内轮花丝基部合生的短雄荵间隔排列, 紧紧围绕 花柱, 使得内轮雄芯花药处于相对稳定的空间位置, 花粉接触传粉者身体的位置也相对固定，提高了传 粉精确性(任明迅, 2008)。

\section{2 雌雄异位与雌雄异熟}

木棉花为柱头探出式雌雄异位, 黄花的雌雄异 位程度比红花更大(图1，图2)。黄花的访花者主要是 蜜蜂, 访花行为随意, 更大的雌雄异位程度有利于 降低䧳雄功能干扰(Webb \& Lloyd，1986) (图1)。

木棉雄性先熟, 但黄花表型的柱头可受性很早就 达到了较高水平，导致黄花雌雄异熟程度小于红花 (图2)。延长的柱头可受性可以提高接收异交花粉的可 能性，这对于极少受到鸟类访问的黄花来说具有一定 的适应意义。另一方面, 在种群内占据数量优势、以 鸟类访问为主的红花，雌雄异熟程度更大，降低了 雌雄功能干扰(Lloyd \& Webb, 1986; 张大勇, 2004)。

因此，木棉红花主要通过雌雄异熟在时间上错 开花药输出花粉(雄性功能)和柱头接受外来花粉 (䧳性功能)的时间，从而避免雌雄功能干扰与自花 授粉; 木棉黄花则主要依赖雌雄异位在空间上错开 花粉输出与接受花粉, 避免了雌雄功能干扰, 并通 过延长雌性阶段(降低雌雄异熟程度)实现一定水平 的异交。

\section{3 黄花个体的适应意义}

木棉的访花者主要为鸟类和蜜蜂(图3)。蜜蜂的 视觉范围在300-600 nm, 鸟类感知的波长为300$700 \mathrm{~nm}$ (Raine \& Chittka, 2007)。因此，红花和黄花 的反射波长均在鸟类视觉范围内; 但蜜蜂对黄色特 别敏感，基本只访问黄花(图3)。

木棉花蜜含糖量较少(表1)。鸟类为了获取更多 的能量, 常需要访问多朵花和多个个体, 有利于异 交授粉。但在自然条件下，木棉红花坐果率仅有 (3.27 \pm 0.93$) \%$ ，存在较大程度的花粉限制。这近似 于印度的木棉红花坐果率(Aluri et al, 2005)。这种花 粉限制现象很可能是由于鸟类访花频率较低、木棉 自交不亲和等因素造成的(Chittka \& Waser, 1997; Aluri et al, 2005)。黄花很少受到鸟类访问，访问黄 花的蜜蜂主要是掠夺花粉的盗粉者(Aluri et al, 2005), 很少实现成功的异交传粉, 因此黄花的坐果 率更低 $(1.08 \pm 0.56) \%$ 。这可能是黄花在种群内发生 频率极低的原因。

但是，黄花个体可能对木棉的繁殖也有着一定 的积极作用。首先，黄花个体也有一定的坐果率， 表明蜜蜂能偶尔实现成功的传粉或鸟类也偶尔访 问黄花，在整体上提高了木棉的繁殖成功机会。其 次, 黄花个体在花开之后柱头很快变为可受, 柱头 
可受性时间较长(图2), 增加了接受花粉的成功率。 此外, 蜜蜂经过长时间的适应, 也能访问红花(Chittka \& Waser, 1997)。因此，在鸟类访花稀缺的情况 下, 黄花吸引来的蜜蜂可能促进红花传粉, 甚至是 红花与黄花之间的传粉，起到传粉保障作用。

Bergamo等(2016)认为，由于鸟类可以同时感 知红花和黄花, 红花并不能直接提高鸟媒吸引力, 更主要的作用是回避了传粉效率较低的蜜蜂的访 问。但蜜蜂经过长时间的适应，也会访问红花(Chittka \& Waser, 1997)。因此, 能够大量吸引蜜蜂的黄 花可能存在另一个适应意义: 黄花的存在减少了蜜 蜂对红花的访问, 避免蜜蜂骚扰鸟类访问红花。

\section{参考文献}

Aluri JSR, Srungavarapu PR, Kone R (2005) Pollination by bats and birds in the obligate outcrosser Bombax ceiba L. (Bombacaceae), a tropical dry season flowering tree species in the Eastern Ghats forests of India. Ornithological Science, 4, 81-87.

APG II (2003) An update of the Angiosperm Phylogeny Group classification for the orders and families of flowering plants: APG II. Botanical Journal of the Linnean Society, 141, 399-436.

Barwick M (2004) Tropical and Subtropical Trees. Timber Press, Portland.

Bergamo PJ, Rech AR, Brito VLG, Sazima M (2016) Flower colour and visitation rates of Costus arabicus support the 'bee avoidance' hypothesis for red-reflecting hummingbird-pollinated flowers. Functional Ecology, 30, 710-720.

Chang SM, Rausher MD (1999) The role of inbreeding depression in maintaining the mixed mating system of the common morning glory, Ipomoea purpurea. Evolution, 53, 1366-1376.

Chittka L, Waser N (1997) Why red flowers are not invisible to bees. Israel Journal of Plant Sciences, 45, 169-183.

Cruden RW (1977) Pollen-ovule ratios: A conservative indicator of breeding systems in flowering plants. Evolution, 35, 964-974.

Dafni A (1992) Pollination Ecology: A Practical Approach. Oxford University Press, Oxford.

Dafni A, Maués MM (1998) A rapid and simple procedure to determine stigma receptivity. Sexual Plant Reproduction, 11, 177-180.

Davis TA, Mariamma KO (1965) Three kinds of stamens in Bombax ceiba L. (Bombacaceae). Botanic Garden Meise, 35, 185-211.

Fenster CB, Armbruster WS, Wilson P, Dudash MR, Thomson JD (2004) Pollination syndromes and floral specialization. Annual Review of Ecology, Evolution, and Systematics, 35, 375-403.

Gigord LDB, Macnair MR, Smithson A (2001) Negative frequency-dependent selection maintains a dramatic flower color polymorphism in the rewardless orchid Dactylorhiza sambucina (L.) Soò. Proceedings of the National Academy of Sciences, USA, 98, 6253-6255.

Gong YB, Huang SQ (2007) On methodology of foraging behavior of pollinating insects. Biodiversity Science, 15,
576-583. (in Chinese with English abstract) [龚燕兵, 黄双 全 (2007) 传粉昆虫行为的研究方法探讨. 生物多样性, 15, 576-583.]

Grunfeld E, Vincent C, Bagnara D (1989) High-performance liquid chromatography analysis of nectar and pollen of strawberry flowers. Journal of Agricultural and Food Chemistry, 37, 290-294.

Irwin RE, Strauss SY, Storz S, Emerson A, Guibert G (2003) The role of herbivores in the maintenance of a flower color polymorphism wild radish. Ecology, 84, 1733-1743.

Joseph N, Siril EA (2013) Floral color polymorphism and reproductive success in annatto (Bixa orellana L.). Tropical Plant Biology, 6, 217-227.

Koski MH, Ashman TL (2016) Macroevolutionary patterns of ultraviolet floral pigmentation explained by geography and associated bioclimatic factors. New Phytologist, 211, 708-718.

Li QL, Ma XK, Cheng J, Luo YB (2012) Quantitative studies of floral color and floral scent. Biodiversity Science, 20, 308-316. (in Chinese with English abstract) [李庆良, 马晓 开, 程瑾, 罗毅波 (2012) 花颜色和花气味的量化研究方 法. 生物多样性, 20, 308-316.]

Majetic CJ, Raguso RA, Ashman T (2009) The sweet smell of success: Floral scent affects pollinator attraction and seed fitness in Hesperis matronalis. Functional Ecology, 23, 480-487.

Raine NE, Chittka L (2007) The adaptive significance of sensory bias in a foraging context: Floral colour preferences in the bumblebee Bombus terrestris. PLoS ONE, 2, e556.

Ren MX (2008) Stamen fusion in plants: Diversity, adaptive significance, and taxonomic implications. Journal of Systematics and Evolution, 46, 452-466. (in Chinese with English abstract) [任明迅 (2008) 植物雄莣合生的多样性、适 应意义及分类学意义初探. 植物分类学报, 46, 452-466.]

Ren MX (2009) Intrafloral stamen differentiations and their adaptive significances. Chinese Journal of Plant Ecology, 33, 222-236. (in Chinese with English abstract) [任明迅 (2009) 花内雄荵分化及其适应意义. 植物生态学报, 33, 222-236.]

Rodriguez-Riano T, Dafni A (2000) A new procedure to assess pollen viability. Sexual Plant Reproduction, 12, 241-244.

Schoonhoven LM, van Loon JJA, Dicke M (2007) Insect-Plant Biology. Oxford University Press, Oxford, UK.

Stanton ML, Snow AA, Handel SN (1986) Floral evolution: Attractiveness to pollinators increases male fitness. Science, 232, 1625-1626.

Vaidya P, Mcdurmon A, Mattoon E, Keefe M, Carley L, Lee CR, Bingham R, Anderson JT (2018) Ecological causes and consequences of flower color polymorphism in a selfpollinating plant (Boechera stricta). New Phytologist, 218, 380-392.

Webb CJ, Lloyd DG (1986) The avoidance of interference between the presentation of pollen and stigmas in angiosperms II. Herkogamy. New Zealand Journal of Botany, 24, 163-178.

Zhang DY (2004) Plant Life-History Evolution and Reproductive Ecology. Science Press, Beijing. (in Chinese) [张大 勇 (2004) 植物生活史进化与繁殖生态学. 科学出版社, 北京.]

Zhou ZL, Ma HC, Lin K, Zhao YJ, Chen Y, Xiong Z, Wang LY, Tian B (2015) RNA-seq reveals complicated transcriptomic responses to drought stress in a nonmodel tropic plant, Bombax ceiba L. Evolutionary Bioinformatics, 11, 27-37. (责任编委：黄双全 责任编辑：时意专) 